\title{
42. RADAR OBSERVATIONS OF THE LEONIDS IN 1965-66
}

\author{
Z. Plavcová \\ (Astronomical Institute of the Czechoslovak Academy of Sciences, Ondrejov)
}

\begin{abstract}
A BSTRACT
Hourly rates of meteor echoes are presented, and it is shown that the peak activity occurred at solar longitude $234^{\circ} .457$ in 1965 , and $234^{\circ} .466$ in 1966 (mean equinox 1950.0). The exponent $s$ is calculated from distribution of echo durations and its variation with date is given.
\end{abstract}

In 1965, the Leonid meteor shower was observed with the Ondřejov meteor radar during 4 days between November 16 and 20. On each day, the observing period was 9 hours around the time of the culmination of the radiant. The antenna beam which is fixed in elevation (directed to $h=45^{\circ}$ ), was steered in azimuth so that it always pointed to the azimuth opposite to that of the radiant. The radiant culminated at 6.30 CET.

Figure 1 shows the hourly rates for each of the 4 days of observations. November 16 is marked by a weak full line, November 17 by a heavy line, November 18 by a dashed line, and November 20 by a dash-dot line. The vertical straight line indicates the time of culmination of the radiant. In the bottom part of the figure are the hourly rates for all echoes. A pronounced maximum occurred on November 17; the smoothed frequencies show a maximum at 5.45 UT, which corresponds to the longitude of the Sun $l=234^{\circ} .457$, mean equinox $1950 \cdot 0$. This time is indicated by a vertical dashed line.

The top part of Figure 1 shows the hourly rates for echoes with duration longer than $10 \mathrm{sec}$. The picture is quite different. In the first half of the observing period, the hourly rates for November 17 do not differ materially from those for November 16; and, curiously enough, in the second part of the observing interval, the activity was higher on November 16 than on November 17. This indicates that the density distribution for bright meteors was different from that of the faint meteors. The peak activity on November 17 is due to medium or faint meteors. This fact is also evident from Figure 2, where we have plotted mean hourly rates averaged over each complete period of observation. The long-enduring echoes fail completely to show the pronounced peak of activity on November 17.

The epoch of maximum activity in 1966 was unfavourable for our geographic longitude, since it occurred when the radiant was already very low in the sky. The radiant sets here at about 13.30 UT. Nevertheless, we attempted to determine the epoch of peak activity. From our diagram in Figure 3 it follows that the maximum

Kresák and Millman (eds.), Physics and Dynamics of Meteors, 432 439. C I.A.U. 
occurred shortly after noon (at 12.03 UT) on November 17 . The corresponding longitude of the Sun is $l=234^{\circ} .466$. This date agrees within 0.009 with the maximum observed by us in 1965 (see above). It is interesting to note that the observations made by Dennis Milon at Kitt Peak indicate a maximum at 11.55 UT, or at $l=234^{\circ} .460$, which gives even better agreement with our observations of 1965 .

One can see at first sight that the Leonids have quite a different distribution of

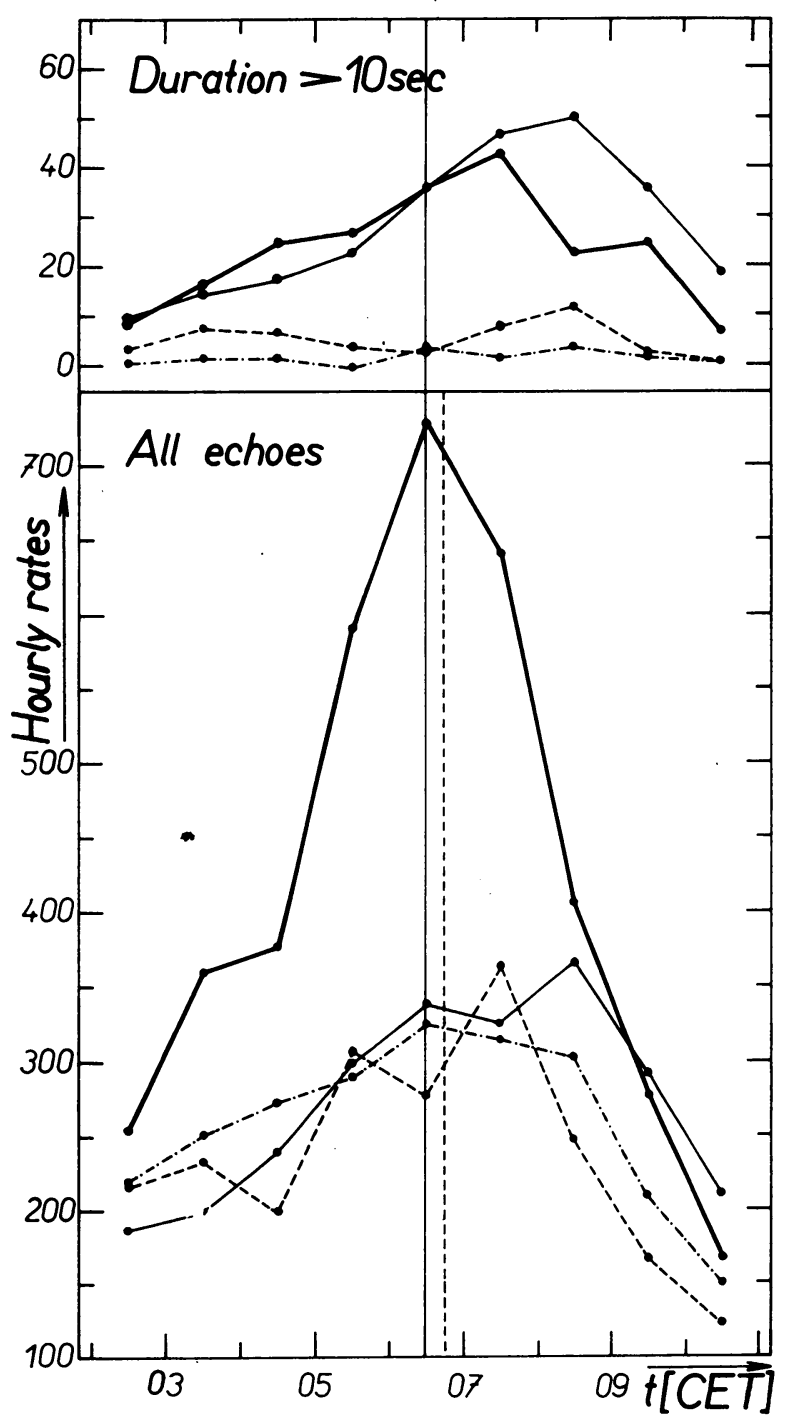

FIG. 1. Hourly rates for November 16,17, 18 and 20,1965. November 16-weak full line, November 17 - heavy line, November 18 -dashed line, November 20 -dashed-and-dotted line. 
masses to the sporadic meteors. This is evident from our statistics of the echo durations. In 1965, the percentage of long-enduring echoes among the Leonids is much higher than among the sporadic meteors and all other meteor showers we have observed so far. Table 1 gives the values of the mass exponent $s$ for each day of observation in 1965 and 1966. All echoes longer than $0.3 \mathrm{sec}$ were used for these statistics. In all cases the value of $s$ was derived by the least-squares method from seven points

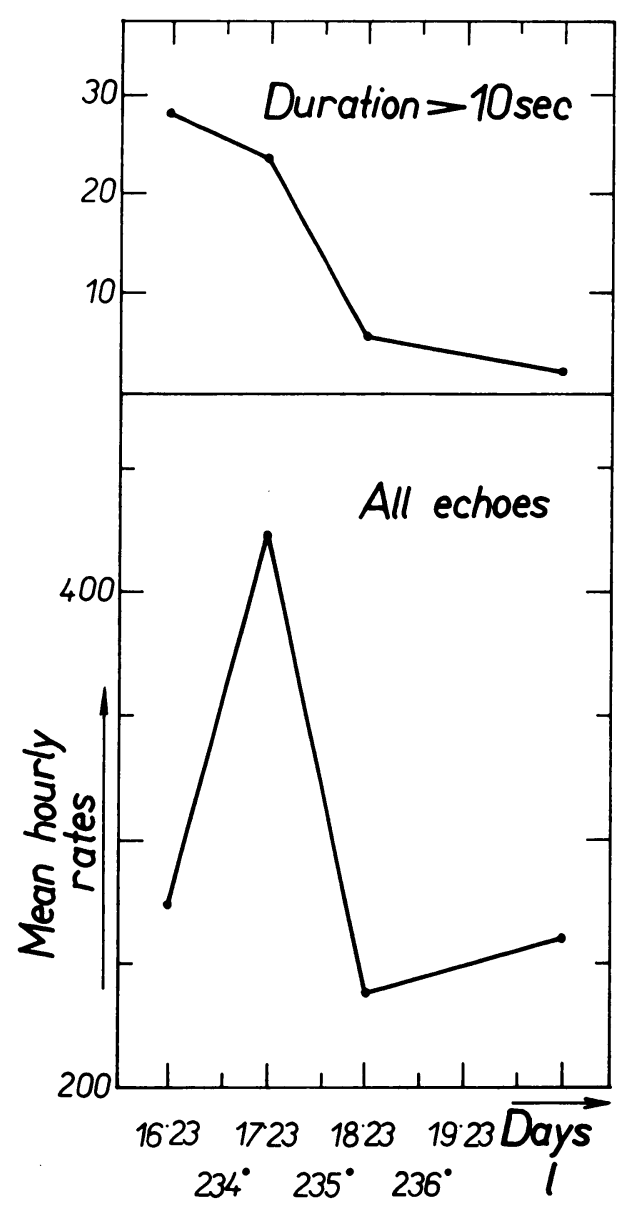

Fig. 2. Mean hourly rates for November 16-20, 1965.

in the diagram of $\log N_{T}$ against $\log T$. Here $T$ is the duration in seconds, and $N_{T}$ is the total number of echoes with duration longer than, or equal to $T$. The highest $T$ used was $6.3 \mathrm{sec}$. For this duration, electron attachment to neutral particles does not significantly affect the duration. It was apparent that longer echoes were affected. 
The interval between 0.4 and $6.3 \mathrm{sec}$ corresponds roughly to meteor magnitudes between $+2 \cdot 5$ and 0 .

The variation of the exponent $s$ with date can be seen in Figure 4, full line; the corresponding values of $\kappa$ are shown by a dashed line. The two lines are actually somewhat affected by the sporadic background; in the lower part of the diagram we give the corrected values.

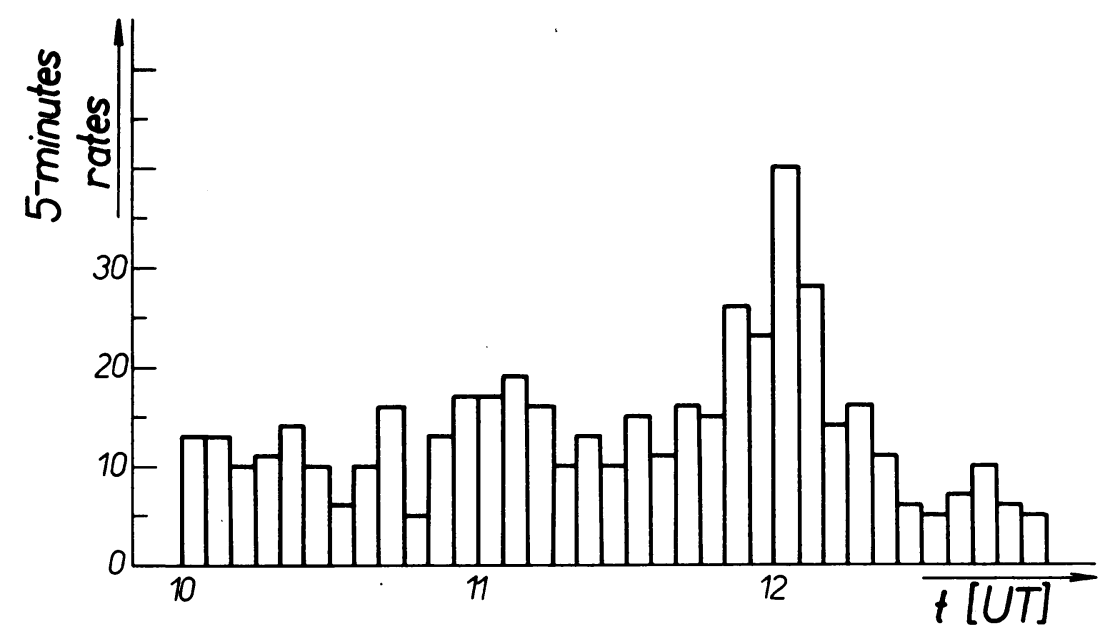

FIG. 3. Five-min rates for November 17, 1966.

Table 1

$\begin{array}{cccc}\text { Year } & \begin{array}{c}\text { Mean longitude } \\ \text { (Mean equintox 1950.0) }\end{array} & \begin{array}{c}s \\ \text { Leonids }+ \text { Non-shower }\end{array} & \begin{array}{c}s \\ \text { Leonids }\end{array} \\ 1965 & 233.437 & 1.28 \pm 0.01 & 1.13 \pm 0.01 \\ & 234.445 & 1.44 \pm 0.01 & 1.35 \pm 0.02 \\ 235.454 & 1.59 \pm 0.02 & \\ 1966 & 237.473 & 1.86 \pm 0.04 & 1.48 \pm 0.05\end{array}$

The sporadic background was eliminated by assuming that the meteor activity on November 20 was purely sporadic, and these echo numbers were subtracted from the data for November 16 and 17. No doubt a few Leonids did appear on November 20, but their contribution was certainly negligible.

On November 16, 1965, the value of $s$ was extremely low, namely $1 \cdot 13$. At the moment of peak activity the value of $s$ was $1 \cdot 35$. Obviously the percentage of bright meteors was unusually high in this stream. This phenomenon may be partly due to the high geocentric velocity of the Leonid meteors. But the main factor must be a 
high percentage of meteors with relatively large masses, otherwise such a phenomenon would be observable with other showers, e.g. the Perseids.

From our observations in 1966, $s$ was computed only from the 3-hour interval of observation between 10 and 13 hours UT on November 17 . We have obtained $s=1 \cdot 61$ for all meteors. After allowance for the sporadic background, the corrected value is $s=1 \cdot 48$. This value is comparable to that for the Geminids (Plavcová, 1965).

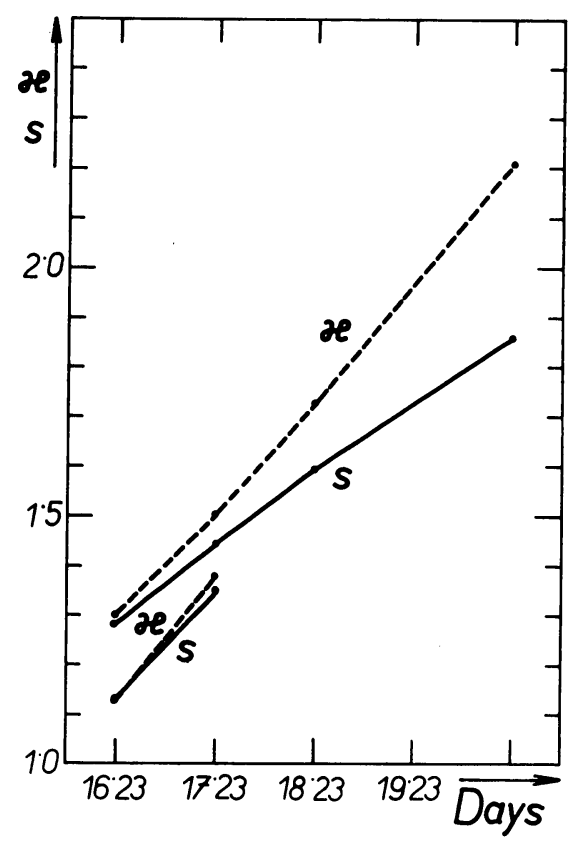

FIG. 4. Variation of $s$ and $\kappa$ for 1965.

\section{Reference}

Plavcová, Z. (1965) Bull. astr. Inst. Csl., 16, 127.

\section{DISCUSSION}

Millman: I wish to comment on the observations of the Leonids, made in Ottawa with the meteor patrol radar during the 1965 and 1966 returns. In a forthcoming paper by McIntosh and myself we show rate plots for Leonid meteors reduced to a radiant elevation of $30^{\circ}$. In 1965 the width of the shower to points one quarter the peak strength was between $1 \frac{1}{2}$ and 2 days, but since the centre of the shower occurred when the radiant was below the horizon at Ottawa, neither the peak rate nor the time of maximum could be accurately determined. In 1966 the width of the shower to quarterstrength points was 1.2 hours and the solar longitude of maximum $234^{\circ} .687$ (1966 epoch). Figures 5 and 6 are taken from the paper by McIntosh and Millman (in press).

McIntosh: For the information of the participants I list in the following table the values of mass 
index $s$ derived from the Ottawa patrol radar observations of recent returns of the Leonid shower

$\begin{array}{ll}1961 & 1.9 \\ 1965, \text { November 16 } & 1.5 \\ 1965 \text {, November 17 } & 1.7 \\ 1966 & 2.2\end{array}$

I estimate the errors as less than $\pm \mathbf{0 \cdot 2}$. Since $s$ was determined by the same method in all cases,

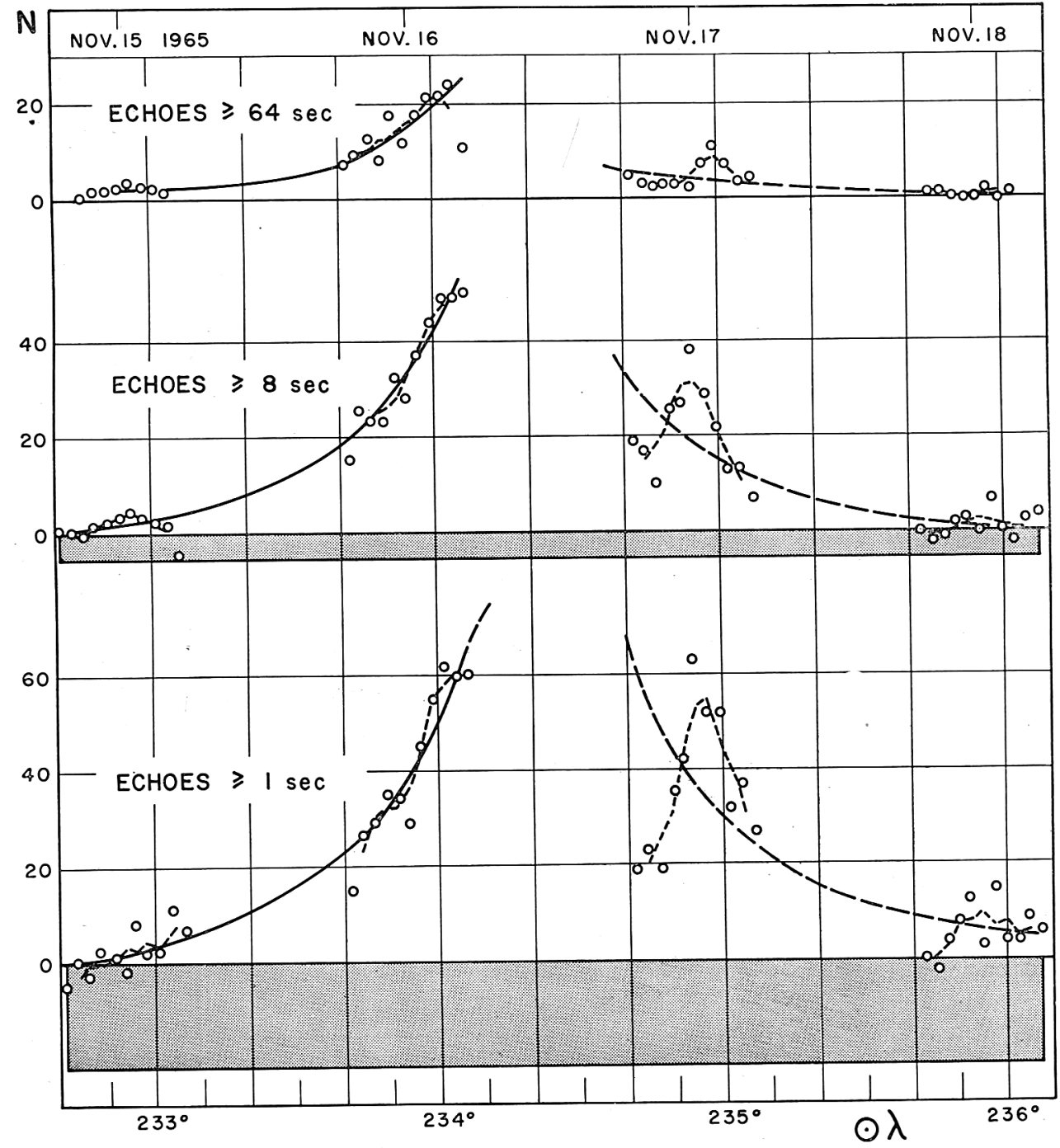

FIG. 5. Hourly rates of Leonid meteors in 1965, recorded with the meteor patrol radar at the Springhill Meteor Observatory near Ottawa. All Leonid rates are reduced to a radiant elevation of $30^{\circ}$. The shaded area represents the mean background hourly rate. 


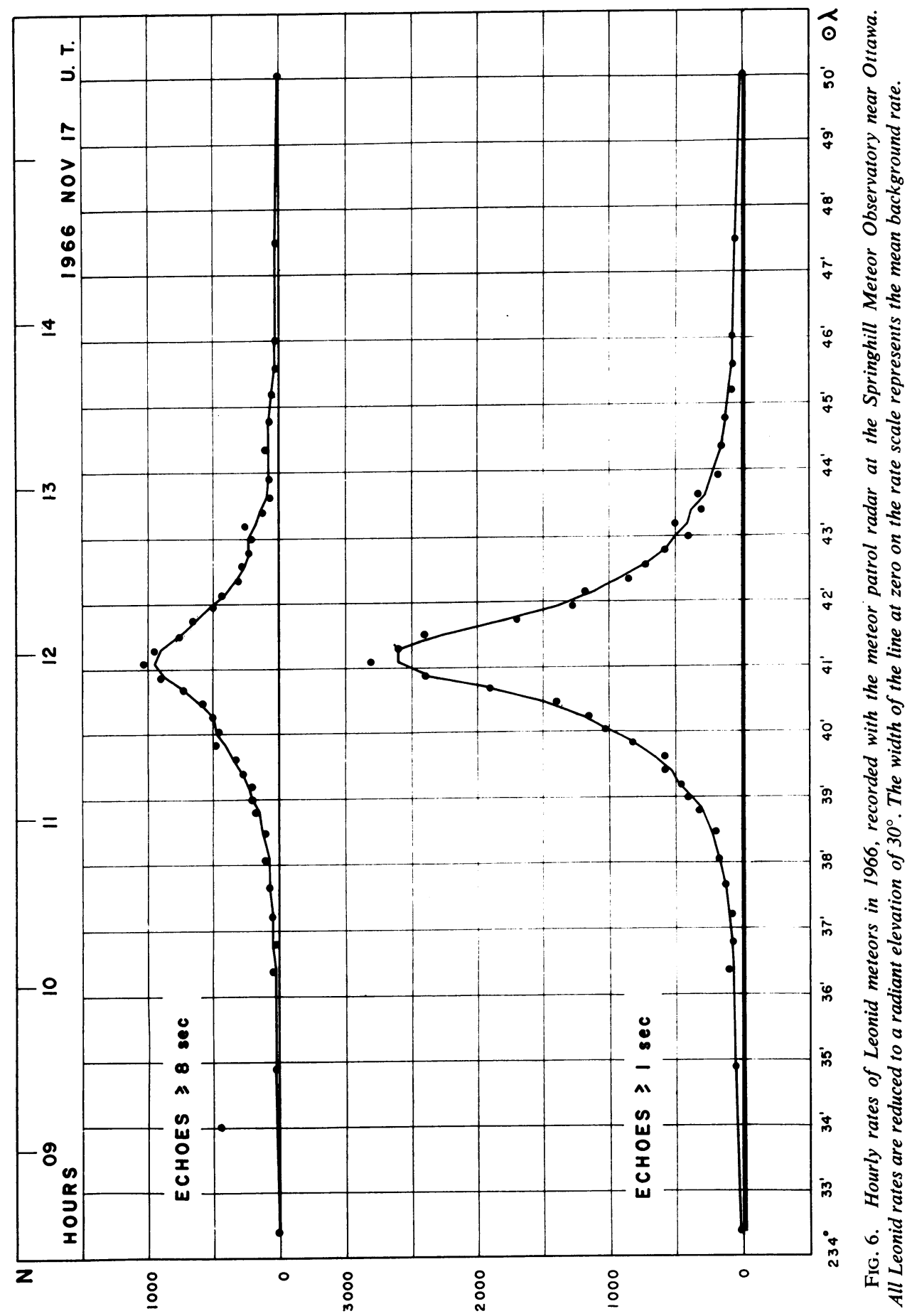


the values should be quite reliable on a relative scale. The variability from year to year is apparent. A second interesting feature concerns total number of echoes observed in 1965 and 1966. Making a reasonable extrapolation of the 1965 rates through the peak value, and finding the areas under the curves, one discovers that the total number of echoes in a count down to 1-sec duration was approximately the same in 1965 and in 1966. From counts down to a higher duration we see that the Earth encountered more large particles passing through the stream in 1965 than in 1966. This result is surprising in view of the high rates in 1966.

Kaiser: I wish to point out that the formulae used for deducing the mass exponent $s$ become inaccurate when $s$ becomes less than about 1.5 . Thus an apparent value of 1.1 has little quantitative significance, it does of course indicate that $s$ is low.

Marsden: I think that the discrepancy of 0.2 between the value of $L_{0}$ given by Mrs. Plavcová and Millman can be explained by precession. Is not the former value referred to equinox 1950 and the latter value to equinox 1965 ? Speaking from memory $I$ believe that the longitude of the ascending node of the orbit of the parent comet Tempel-Tuttle is in very close agreement with $L_{0}$ at the maximum Leonid activity.

Guth: The equinox used by Plavcová is 1950.0 and that explains the discrepancy.

Dohnanyi: It can be shown that the $s$ value for showers should decrease in time beginning at the small mass limit (of the distribution) provided that the shower is no longer being replenished by fresh particles. It therefore follows that unusually small $s$ values may indicate that the portion of the shower under observation is relatively 'old' (provided that the initial $s$ value was higher).

Whipple: Along the same lines I was thinking that one should look for an increase of radiant spread with decrease in the mass index. Collisions and erosion should increase the scatter in space motion for particles in an older depleted stream.

Kresák: If I remember correctly, there are some visual and telescopic observations of the Leonids from the early 'thirties, published by Watson, which indicate an abrupt change of the magnitude function at about $+2^{\mathrm{m}}$, with $s$ dropping down to about $1 \cdot 0$. 